This item was submitted to Loughborough's Research Repository by the author.

Items in Figshare are protected by copyright, with all rights reserved, unless otherwise indicated.

\title{
Continued fractions and solutions of the Feigenbaum-Cvitanovic equation
}

PLEASE CITE THE PUBLISHED VERSION

\section{LICENCE}

CC BY-NC-ND 4.0

\section{REPOSITORY RECORD}

Mestel, B.D., Andrew H. Osbaldestin, and Alexei V. Tsygvintsev. 2019. "Continued Fractions and Solutions of the Feigenbaum-cvitanovic Equation”. figshare. https://hdl.handle.net/2134/438. 


\title{
Continued fractions and solutions of the Feigenbaum-Cvitanović equation
}

\author{
B.D.Mestel ${ }^{\mathrm{a}}$ A.H. Osbaldestin ${ }^{\mathrm{b}}$ A.V. Tsygvintsev ${ }^{\mathrm{b}}$ \\ a School of Mathematical Sciences \\ University of Exeter, Exeter, EX4 4QE, UK E-mail: B.D.Mestel@ex.ac.uk \\ ${ }^{\mathrm{b}}$ Department of Mathematical Sciences \\ Loughborough University, Loughborough, LE11 3TU, UK \\ E-mail: A.H.Osbaldestin@lboro.ac.uk, A.Tsygvintsev@lboro.ac.uk
}

January, 2002

\begin{abstract}
In this paper, we develop a new approach to the construction of solutions of the Feigenbaum-Cvitanović equation whose existence was shown by H. Epstein. Our main tool is the analytic theory of continued fractions.
\end{abstract}

\section{Introduction}

In the present paper we consider the Feigenbaum-Cvitanović functional equation

$$
g(x)=-\lambda^{-1} g(g(\lambda x)), \quad g(0)=1, \quad-g(1)=\lambda \in(0,1),
$$

where $g$ is a map of the interval $[-1,1]$ into itself. We only consider solutions $g$ such that, on $[0,1), g(x)=F\left(x^{d}\right), d>1$, with $F$ analytic, decreasing, and without critical points. Let $U=F^{-1}$ be the inverse function of $F$. Then $U$ will satisfy

$$
U\left(U(-\lambda x)^{1 / d}\right)=\lambda U(x), \quad U(-\lambda)=1, \quad U^{\prime}(-\lambda)=-d \lambda^{d-1} .
$$

We denote $\mathbb{C}_{+}=-\mathbb{C}_{-}=\{\zeta \in \mathbb{C}, \operatorname{Im} \zeta>0\}$.

Theorem 1.1 (Epstein, 1986). The solution $U$ of the equation (1.2) exists for all $d>1$ and extends to a function holomorphic in $\mathbb{C}_{+} \cup \mathbb{C}_{-} \cup\left(-\lambda^{-1}, \lambda^{-2}\right)$ which is injective there and $U\left(\mathbb{C}_{+}\right) \subset \mathbb{C}_{-}, U\left(\mathbb{C}_{-}\right) \subset \mathbb{C}_{+}$.

An analytic function $f$ which maps $\mathbb{C}_{+}$into $\mathbb{C}_{+}$and $\mathbb{C}_{-}$into $\mathbb{C}_{-}$is called a Herglotz function ( $-f$ is an anti-Herglotz function ). See [1] for details.

In the proof of Theorem 1.1 Epstein used in an essential way the integral representation for anti-Herglotz functions, which provides us with a priori bounds of the form $r(x) \leq$ $U(x) \leq R(x)$ where $r(x), R(x)$ are known rational functions (see [2], [3], [4]). These bounds contain some initial basic information about the behaviour of $U$.

The purpose of the present paper is to make more precise the analytic nature of the solution of the functional equation (1.2) and give some new rational bounds on it. Our main tool here is the analytic theory of continued fractions, a detailed account of which may be 
found in the book by Wall [5]. The foundation of this theory is the natural correspondence between continued fractions, analytic functions and their integral representation given by a moment problem. For concreteness, let us consider the Stieltjes integral of the form

$$
f(z)=\int_{0}^{1} \frac{d \theta(u)}{1+u z}, \quad \int_{0}^{1} d \theta(u)=1,
$$

where $\theta(u)$ is a bounded nondecreasing function in $(0,1)$. Then $f$ has the continued fraction representation $[5]$

$$
f(z)=\frac{1}{1+\frac{g_{1} z}{1+\frac{\left(1-g_{1}\right) g_{2} z}{1+\cdots}}}, \text { denoted by } f(z)=\left\{g_{1}, g_{2}, \cdots \mid z\right\} \text {, }
$$

where the real coefficients $g_{p} \in[0,1], p=1,2, \ldots$ are certain rational functions of the moments $\mu_{p}$ of $\theta(u)$ defined by $\mu_{p}=\int_{0}^{1} u^{p} d \theta(u), p \geq 1$. In Section 2 we show that the function $u=U^{1 / d}$, where $U$ is a solution of (1.2), always can be reduced, by a certain conformal change, to the form (1.4). In Section 3 we derive a new uniform bound on $\lambda^{d}$ for $d \geq 2$ which constitutes the main result of the present paper. Section 4 contains some numerical results.

\section{Wall functions and their continued fraction repre- sentation}

Denote by $\mathcal{D}$ the portion of the complex plane $\mathbb{C}$ exterior to the cut along the real axis from $-\infty$ to -1 . Let $f(z)$ satisfying $f(0)=1$, be an anti-Herglotz function analytic in $\mathcal{D}$ and positive for $z>-1$. We call $f$ a Wall function if its square $f^{2}$ is again an anti-Herglotz function. Let $\mathcal{W}$ be the set of all Wall functions.

Theorem 2.1. For an arbitrary $f \in \mathcal{W}$ we have $f(z)=\left\{g_{1}, g_{2}, \cdots \mid z\right\}$ for some uniquely defined sequence of coefficients $g_{i} \in[0,1], i \geq 1$. The corresponding continued fraction converges uniformly on every compact domain in $\mathcal{D}$.

Proof. Let $f \in \mathcal{W}$ then from the definition of a Wall function it follows that $f$ is an antiHerglotz function which is analytic in the domain $\mathcal{D}$, real for real values of $z$ and having a positive real part. Consider the function $\phi=\sqrt{1+z}$ where the square root is positive on the axis $(-1,+\infty)$. The function so obtained is a Herglotz function since the argument of $\phi$ is one half the argument of $1+z$. We will check that the product $\Phi=\phi f$ is a function which is analytic in the domain $\mathcal{D}$, real for real values of $z$ and having a positive real part. Let $f=f_{1}+i f_{2}, \phi=\phi_{1}+i \phi_{2}$ where $f_{1}, \phi_{1}$ and $f_{2}, \phi_{2}$ are the corresponding real and imaginary parts of the functions $f$ and $\phi$. Then $\operatorname{Re}(\Phi)=\phi_{1} f_{1}-\phi_{2} f_{2}>0$ in $\mathcal{D}$. Indeed, $\phi_{1} f_{1}>0$ since both $f$ and $\phi$ have positive real parts and $-\phi_{2} f_{2} \leq 0$ since the imaginary parts of $f, \phi$ always have a different sign in $\mathcal{D}$. By the theorem of Wall [5, p. 279] the function 
$\Phi=\phi f$ can be represented in the form $\Phi=\sqrt{1+z}\left\{g_{1}, g_{2}, \cdots \mid z\right\}$ for some uniquely defined sequence $g_{i} \in[0,1], i \geq 1$, where the corresponding continued fraction converges uniformly on every compact domain in $\mathcal{D}$. Cancelling $\sqrt{1+z}$, it follows that $f$ is of the form (1.4). This concludes the proof.

Remark 1. It is easy to see from the integral representation (1.3) that $f(z)=\left\{g_{1}, g_{2}, \cdots \mid z\right\}$ is an anti-Herglotz function.

We will use the following notation for partial approximants of (1.4)

$$
\left\{g_{1}, \ldots, g_{k} \mid z\right\}=\frac{1}{1+\frac{g_{1} z}{1+\frac{\left(1-g_{1}\right) g_{2} z}{1+\frac{\cdots}{1+\left(1-g_{k-1}\right) g_{k} z}}}}, \quad k \geq 1,
$$

which are all rational anti-Herglotz functions.

The continued fraction $f(z)=\left\{g_{1}, g_{2}, \cdots \mid z\right\}$ can be approximated by rational functions as given by the following theorem

Theorem 2.2. a) Let $k=2 n+1, n=0,1,2, \ldots$, then

$$
A_{k}(z) \leq f(z) \leq B_{k}(z), \quad-1<z<+\infty,
$$

where $A_{k}(z)=\left\{g_{1}, \ldots, g_{k} \mid z\right\}, \quad B_{k}(z)=\left\{g_{1}, \ldots, g_{k}, 1 \mid z\right\}$

b) Let $k=2 n, n=1,2, \ldots$, then

$$
A_{k}^{+}(z) \leq f(z) \leq B_{k}^{+}(z), \quad 0 \leq z<+\infty \quad \text { and } \quad A_{k}^{-}(z) \leq f(z) \leq B_{k}^{-}(z), \quad-1<z<0,
$$

where $A_{k}^{+}(z)=\left\{g_{1}, \ldots, g_{k}, 1 \mid z\right\}, \quad B_{k}^{+}(z)=\left\{g_{1}, \ldots, g_{k} \mid z\right\}$ and $A_{k}^{-}(z)=B_{k}^{+}(z), B_{k}^{-}(z)=$ $A_{k}^{+}(z)$.

The proof is based on Theorems 1.11 and Theorems 14.2-14.3 of [5] and is straightforward

Applying Theorem 2.2 in the case $k=1$ we obtain $A_{1}(z) \leq f(z) \leq B_{1}(z), z \in(-1, \infty)$ where

$$
A_{1}(z)=\left(1+g_{1} z\right)^{-1}, \quad B_{1}(z)=\left(1+\left(1-g_{1}\right) z\right)(1+z)^{-1}
$$

\section{Applications to the Feigenbaum-Cvitanović- equa- tion}

In this section we fix $U$ to be a solution of the functional equation (1.2) given by Theorem 1.1 for a certain $d>1$. Then the equation (1.2) written in terms of the function $u=U^{1 / d}$ 
takes the form

$$
u(u(-\lambda z))=\lambda u(z), \quad \lambda \in(0,1),
$$

where $u(z)=U(z)^{1 / d}$ satisfies the conditions $u(1)=0, \quad u(-\lambda)=1, \quad u^{\prime}(-\lambda)=-\lambda^{d-1}$ from (1.2) and is an anti-Herglotz function analytic in the domain $\mathbb{C}_{+} \cup \mathbb{C}_{-} \cup\left(-\lambda^{-1}, 1\right)$.

Let us consider the conformal mapping $f_{\lambda}(z)=(1-\lambda)^{-1}(\lambda+z)(1-z)^{-1}$ which sends the real interval $\left(-\lambda^{-1}, 1\right)$ into $(-1,+\infty)$ bijectively and satisfies $f_{\lambda}(-\lambda)=0, \quad f_{\lambda}\left(-\lambda^{-1}\right)=-1$. Note that both $f_{\lambda}$ and $f_{\lambda}^{-1}$ are Herglotz functions. Then $u \circ f_{\lambda}^{-1} \in \mathcal{W}$ for an arbitrary $d \geq 2$ which proves the following proposition

Proposition 3.1. Let $u(z)=U(z)^{1 / d}$ be the solution of the functional equation (3.1) with corresponding parameters $d \geq 2, \lambda \in(0,1)$. Then $u(z)=\left\{g_{1}, g_{2}, \cdots \mid f_{\lambda}(z)\right\}$ with certain uniquely defined coefficients $g_{k} \in[0,1], k \geq 1$.

It turns out that $g_{1}$ depends only on the numbers $\lambda$ and $d$. In fact, using the condition $u^{\prime}(-\lambda)=-\lambda^{d-1}$, by a simple differentiation of $u$ we obtain

$$
g_{1}=\lambda^{d-1}\left(1-\lambda^{2}\right)
$$

Using the rational bounds given by Theorem 2.2 it is easy to write the corresponding bounds on the function $u(z): A\left(f_{\lambda}(z)\right) \leq u(z) \leq B\left(f_{\lambda}(z)\right), z \in\left(-\lambda^{-1}, 1\right)$ where $A(z), B(z)$ are the lower and upper bounds given by formulas (2.1)-(2.2) for a certain $k \geq 1$. Let us denote $\mathcal{A}(z)=A\left(f_{\lambda}(z)\right), \mathcal{B}(z)=B\left(f_{\lambda}(z)\right)$ then we have $u(-\lambda z) \in[\mathcal{A}(-\lambda z), \mathcal{B}(-\lambda z)]$ and $u(u(-\lambda z)) \in[\mathcal{A}(\mathcal{B}(-\lambda z)), \mathcal{B}(\mathcal{A}(-\lambda z))]$ for $z \in\left(-\lambda^{-1}, 1\right)$. Thus, if $u$ satisfies the equation (3.1) then the following inequalities must be satisfied

$$
\mathcal{B}(\mathcal{A}(-\lambda z)) \geq \lambda \mathcal{A}(z), \quad \mathcal{A}(\mathcal{B}(-\lambda z)) \leq \lambda \mathcal{B}(z), \quad \forall z \in\left(-\lambda^{-1}, 1\right)
$$

The rational functions $\mathcal{A}(z), \mathcal{B}(z)$ contain $g_{1}, \ldots, g_{k}, \lambda$ as parameters so that (3.3) imposes certain restrictions on these numbers and can be used together with (3.2) to obtain relations between $g_{i}, \lambda$ and $d$. The degree of accuracy will depend essentially on the number $k$ but even for small $k$ this method gives satisfactory results. We consider the simplest case $k=1$. Taking $\mathcal{A}(z), \mathcal{B}(z)$ corresponding to bounds given by (2.3) and using the first inequality from (3.3) for $z \rightarrow-\lambda^{-1}$, we obtain

$$
\mathcal{B}(0) \geq \lambda \mathcal{A}\left(-\lambda^{-1}\right) \quad \text { or } \quad 1-\lambda+\left(1-g_{1}\right) \lambda \geq \lambda\left(1-g_{1}\right)^{-1}
$$

which implies

$$
0 \leq g_{1} \leq \theta(\lambda), \quad \text { where } \quad \theta(\lambda)=\lambda^{-1}\left(1+\lambda-\sqrt{1-2 \lambda+5 \lambda^{2}}\right) / 2 .
$$

Using (3.2), this gives the following lower bound on $d$

$$
d \geq \log \left(\frac{\lambda \theta(\lambda)}{1-\lambda^{2}}\right) / \log (\lambda), \quad d \geq 2
$$


The inequality (3.4) can be written in the form $\lambda^{d} \leq \zeta(\lambda), \zeta(\lambda)=\lambda \theta(\lambda) /\left(1-\lambda^{2}\right)$. It is easy to show that $\zeta^{\prime}(\lambda)$ vanishes at a unique point $\lambda_{c}$ in $(0,1)$ and $\zeta^{\prime \prime}\left(\lambda_{c}\right)<0$. Hence in this interval $\zeta$ has just one maximum. From this we derive the following uniform bound

$$
\lambda^{d} \leq c, \quad c=0.26308 \ldots, \quad \text { for } \quad d \geq 2,
$$

where we omit the explicit expression for $c$ (which is an algebraic number) due to its complexity. We believe that a more careful analysis of inequalities (3.3) for $k>1$ can considerably improve this estimate.

\section{Numerical applications}

In this section we will discuss briefly a numerical technique based on the application of Proposition 3.1. We fix $d \geq 2$ and look for a rational approximation of the solution $u(z)$ of the equation (3.1) in the form $r(z)=\left\{g_{1}, \ldots, g_{n} \mid f_{\lambda_{a}}(z)\right\}, n>1$ with $g_{1}=\lambda_{a}^{d-1}\left(1-\lambda_{a}^{2}\right)$ for a certain sequence of real numbers $g_{k} \in[0,1]$ and $\lambda_{a} \in \mathbb{R}$. One can show that the substituting of $r(z)$ into (3.1) with $\lambda=\lambda_{a}$ gives us the new function $R(z)=\left\{\tilde{g}_{1}, \tilde{g}_{2}, \cdots \mid f_{\lambda_{a}}(z)\right\}$. The numbers $\tilde{g}_{k}$ are not necessary in the interval $[0,1]$ and are certain functions of $\lambda_{a}, g_{1}, \ldots, g_{n}$. Let us suppose that the first $n$ coefficients of the continued fraction representations of $r(z)$ and $R(z)$ are the same i.e. $g_{i}=\tilde{g}_{i}, 1 \leq n \leq n$. This system of equations can be solved using, for example, Newton's method. Numerical experiments show that it seems to have a solution for all $d \geq 2$ and $n>1$ with $\lambda_{a}, g_{1}, \ldots, g_{n}$ belonging to the interval [0,1] which is as expected in view of our Proposition 2.1. Since $r(z)$ is a rational anti-Herglotz function, it can be always be represented in the simple form $r(z)=c+\sum_{i=1}^{K} \frac{r_{i}}{z-z_{i}}$ with some $c, z_{i} \in \mathbb{R}$ and $r_{i} \in \mathbb{R}_{+}$. In the classical Feigenbaum case, $d=2$, this method gives, for $n=7$, the following rational approximation for $u(z)$

$$
u(z)=2.822306+\frac{1.144796}{z+4.06181}+\frac{0.003406}{z-1.021068}+\frac{0.139511}{z-1.373872}++\frac{15.718028}{z-7.253131},
$$

with the corresponding value $\lambda_{a}=0.399778 \ldots$ and $g_{i}$ given by

$$
g_{1}=0.3359, g_{2}=0.2779, g_{3}=0.2951, g_{4}=0.3980, g_{5}=0.5023, g_{6}=0.2368, g_{7}=0.0277
$$

The value of $\lambda_{a}$ is in a good correspondence with the val,ue of $\lambda=0.399535 \ldots$ for the families of quadratic maps, known from the classical theory of Feigenbaum. For $r(z)$ given by (4.1) we have $\max |r(z)-R(z)|<6 \times 10^{-7}$ for $-\lambda_{a} \leq z \leq 0$ and $\max |r(z)-R(z)|<5 \times 10^{-2}$ for $0 \leq z \leq 1$.

\section{References}

[1] Donoghue, William F., Jr. Monotone matrix functions and analytic continuation, Die Grundlehren der mathematischen Wissenschaften, Band 207, Springer-Verlag, New York-Heidelberg, 1974

[2] Epstein, H. New proofs of the existence of the Feigenbaum functions, Comm. Math. Phys. 106 (1986), no. 3, 395-426 
[3] Epstein, H. Fixed points of composition operators, Procceedings of a NATO Advanced Study Institute on Nonlilenar Evolution, Italy, 1987, 71-100

[4] Epstein H., Lascoux J. Analyticity properties of the Feigenbaum function, Commun. Math. Phys. 81, 1981, 437-453,

[5] Wall, H.S. Analytic theory of continued fractions, D. Van Nostrand Company, Inc., New York, N.Y., 1948 